\title{
INDEX TO VOLUME 69
}

\section{RESEARCH ANNOUNCEMENTS}

Aanderaa, Stål. See Dreben, Burton.

Abraham, Ralph. Transversality in manifolds of mappings, 470.

Anderson, R. D. On raising flows and mappings, 259.

Andrews, Peter. See Dreben, Burton.

Aronson, D. G. On the Green's function for second order parabolic differential equations with discontinuous coefficients, 841.

Atiyah, M. F. and Singer, I. M. The index of elliptic operators on compact manifolds, 422.

Atkinson, F. V. Boundary problems leading to orthogonal polynomials in several variables, 345.

Auslander, Louis. Complete locally affine spaces and algebraic hulls of matrix groups, 242.

Auslander, L. and Green, L. Flows on solvmanifolds, 745.

Baum, L. E., Ferguson, J. D. and Katz, Melvin. Infinitely repeated matrix games for which pure strategies suffice, 467.

Baum, L. E. and Katz, Melvin. Convergence rates in the law of large numbers, 771.

Baum, P. F. Cohomology of homogeneous spaces, 531.

Blum, J. R. and Hanson, D. L. On the isomorphism problem for Bernoulli schemes, 221.

Boehme, T. K. On approximate solutions to the convolution equation on the half-line, 847.

de Branges, Louis. Invariant subspaces of nonselfadjoint transformation, 587.

Brauer, George. Convolution of sequences, 216.

Browder, F. E. Nonlinear elliptic boundary value problems, 862.

. Nonlinear parabolic boundary value problems of arbitrary order, 858.

Brown, E. H., Jr. and Peterson, F. P. Relations between Stiefel-Whitney classes of manifolds, 228.

Brown, Morton and Gluck, Herman. Stable structures on manifolds, 51.

Brumer, Armand. Structure of hereditary orders, 721.

Buchsbaum, D. A. and Rim, D. S. A generalized Koszul complex, 382.

Cantor, D. G. Power series with integral coefficients, 362.

Cantrell, J. C. Almost locally flat embeddings of $S^{n-1}$ in $S^{n}, 716$.

Charlap, L. S. and Vasquez, A. T. The cohomology of group extensions, 815.

Chen, Kuo-Tsai. On local diffeomorphisms about an elementary fixed point, 838.

$\mathrm{Chu}, \mathrm{Hsin}$ and Geraghty, M. A. The fundamental group and the first cohomology group of a minimal set, 377.

Cohen, Eckford. An elementary estimate for the $k$-free integers, 762.

Cohen, P. J. A minimal model for set theory, 537.

Connell, E. H. Stable homeomorphisms can be approximated by piecewise linear ones, 87.

Conner, P. E. A bordism theory for actions of an Abelian group, 244.

Pontrjagin numbers of maps, 276.

Crawley, Peter and Jonsson, Bjarni. Direct decompositions of algebraic systems, 541.

Cross, J. T. The number of solutions of a trinomial congruence involving a kth power and a square, 83.

Cudia, D. F. On the localization and directionalization of uniform convexity, 265.

Davis, Morton and Maschler, Michael. Existence of stable payoff configurations for cooperative games, 106.

Dembowski, Peter. Inversive planes of even order, 850. 
Diaz, J. B. and Metcalf, F. T. Stronger forms of a class of inequalities of G. Polya-G. Szegö and L. V. Kantorovich, 415.

Dreben, Burton, Andrews, Peter and Aanderaa, Stål. False lemmas in Herbrand, 699.

Dubins, L. E. and Freedman, D. A. Random distribution functions, 548.

Fan, Ky. Invariant subspaces of certain linear operators, 773.

Feldman, Edgar. The geometry of immersions. I, 693.

Ferguson, J. D. See Baum, L. E.

Freedman, D. A. See Dubins, L. E.

Fuller, F. B. Periodic trajectories of a one-parameter semigroup, 409.

Gangolli, Ramesh. Isotropic infinitely divisible processes on compact symmetric spaces, 357.

Geraghty, M. A. See Chu, Hsin.

Gil de Lamadrid, Jesás. Uniform cross norms and tensor products of Banach algebras, 797.

Glicksberg, I. See de Leeuw, K.

Gluck, Herman. Restriction of isotopies, 78.

Unknotting $S^{1}$ in $S^{4}, 91$.

Embeddings in the trivial range, 824.

See Brown, Morton.

Gosselin, R. P. Integral norms of subadditive functions, 255.

Granirer, E. On left amenable semigroups which admit countable left invariant means, 101.

Grant, K. and Whaples, G. Correction to "Abstract class formations," 123.

Gray, Alfred and Shah, S. M. A note on entire functions and a conjecture of Erdös, 573.

Greathouse, Charles. Locally fat, locally tame, and tame embeddings, 820.

Green, L. See Auslander, L.

Greenberg, Leon. Maximal Fuchsian groups, 569.

Gustin, William. Orientable embedding of Cayley graphs, 272.

Hanson, D. L. A representation theorem for stationary Markov chains, 465. See Blum, J. R.

Harish-Chandra. Invariant eigendistributions on semisimple Lie groups, 117.

Hayes, David. A polynomial analog of the Goldbach conjecture, 115.

Correction to "A polynomial analog of the Goldbach conjecture," 493.

Heins, Maurice. Fundamental polygons, 747.

Helgason, S. Duality and random transform for symmetric spaces, 782. 778.

Helms, L. L. Maximal wedges of subharmonic functions, 710.

See Johnson, Guy.

Hillman, Abraham. See Lowan, A. N.

Hirsch, M. W. Obstruction theories for smoothing manifolds and maps, 352.

Hsiang, W. C. and Szczarba, R. H. On the embeddability and nonembeddability of certain parallelizable manifolds, 534 .

Ingelstam, Lars. Hilbert algebras with identity, 794.

James, I. M. On the immersion problem for real projective spaces, 231.

Johnson, Guy and Helms, L. L. Class D supermartingales, 59.

Jones, B. F., Jr. Singular integrals and parabolic equations, 501.

Jonsson, Bjarni, See Crawley, Peter.

Kakutani, S. and Parry, W. Infinite measure preserving transformations with "mixing," 752. 
Katz, Melvin. See Baum, L. E.

Kelman, R. B. Axisymmetric potential problems suggested by biological considerations, 835.

Kingman, J. F. C. A continuous time analogue of the theory of recurrent events, 268.

Kister, J. M. Microbundles are fibre bundles, 854.

Klingenberg, Wilhelm. On the topology of Riemannian manifolds where the conjugate points have a similar distribution as in symmetric spaces of rank 1, 95.

Korányi, Adam. On the boundary values of holomorphic functions in wedge domains, 475.

Kostant, Bertram. Lie group representations on polynomial rings, 518.

Kunita, Hiroshi and Watanabe, Takesi. Markov processes and Martin boundaries, 386.

deLeeuw, K. and Glicksberg, I. Analytic measures on compact groups, 46.

Levine, J. On differentiable imbeddings of simply-connected manifolds, 806.

Lewittes, Joseph. Gaps at Weierstrass points for the modular group, 578.

Lima, E. L. Commuting vector fields on 2-manifolds, 366.

Lindenstrauss, Joram. Some results on the extension of operators, 582.

Littman, Walter. Fourier transforms of surface-carried measures and differentiability of surface averages, 766.

Lowan, A. N., Salzer, H. E. and Hillman, Abraham. Errata to "A table of coefficients for numerical differentiation," 874.

McKissick, Robert. A nontrivial normal sup norm algebra, 391.

MacNerney, J. S. An integration-by-parts formula, 803.

Marcus, Marvin. The permanent analogue of the Hadamard determinant theorem, 494.

Maschler, Michael. See Davis, Morton.

Maskit, Bernard. On a conjecture concerning planar coverings of surfaces, 396.

Metcalf, F. T. See Diaz, J. B.

Michael, E. The product of a normal space and a metric space need not be normal, 375.

Minc, Henryk. Upper bounds for permanents of $(0,1)$-matrices, 789.

Minty, G. J. Two theorems on nonlinear functional equations in Hilbert space, 691.

Nerode, A. A decision method for p-adic integral zeros of diophantine equations, 513.

Neuwirth, Lee. A topological classification of certain 3-manifolds, 272.

Newman, M. Normal congruence subgroups of the $t \times t$ modular group, 719.

Oehmke, R. H. and Sandler, Reuben. The collineation groups of division ring planes. $\mathrm{I}$. Jordan algebras, 791.

Papakyriakopoulos, C. D. A reduction of the Poincare conjecture to other conjectures. II, 399.

Parry, W. See Kakutani, S.

Peleg, Bezalel. Existence theorem for the bargaining set $\mathbf{M}_{1}^{(i)}, 109$.

Peterson, F. P. See Brown, E. H., Jr.

Petrich, Mario. The maximal semilattice decomposition of a semigroup, 342.

Prosser, R. T. On the consistency of quantum field theory, 552.

Pukanszky, Lajos. On the Plancherel theorem of the $2 \times 2$ real unimodular group, 504.

Putnam, C. R. On the structure of semi-normal operators, 818.

Rao, M. M. Some inference theorems in stochastic processes, 72.

Rapaport, E. S. On a problem of Papakyriakopoulos, 402.

Redheffer, R. M. Differential inequalities, 239.

Uniqueness, stability and error estimation, 497.

Rieffel, M. A. A characterization of commutative group algebras and measure algebras, 812.

Rieger, G. J. On linked binary representations of pairs of integers: some theorems of the Romanov type, 558. 
Rim, D. S. See Buchsbaum, D. A.

Rosen, R. H. Polyhedral neighborhoods in triangulated manifolds, 359.

Rothaus, O. S. The construction of homogeneous convex cones, 248.

Rubel, L. A. and Shields, A. L. Bounded approximation by polynomials, 591.

Rudin, Walter. Idempotents in group algebras, 224.

Salzer, H. E. See Lowan, A. N.

Sandler, Reuben. See Oehmke, R. H.

Sarason, Donald. Doubly invariant subspaces of annulus operators, 593.

Sato, Daihachiro. On the rate of growth of entire functions of fast growth, 411.

Schmidt, W. M. Remark on my paper "Simultaneous approximation and algebraic independence of numbers," 255.

Semadeni, Z. Free and direct objects, 63.

Serrin, James. A Harnack inequality for nonlinear equations, 481.

Shah, S. M. See Gray, Alfred.

Shields, A. L. See Rubel, L. A.

Singer, I. M. See Atiyah, M. F.

Slowikowski, W. On the continuity of inverse operators in (LF)-spaces, 832.

Smith, P. A. The cohomology of certain orbit spaces, 563.

Springer, George. Fredholm eigenvalues and quasiconformal mapping, 810.

Srinivasan, T. P. Simply invariant subspaces, 706.

Stoller, Gerald. Example of a proper subgroup of $S_{\infty}$ which has a set-transitivity property, 220.

Stout, E. L. Two theorems concerning functions holomorphic on multiply connected domains, 527.

Szczarba, R. H. See Hsiang, W. C.

Taibleson, M. H. Lipschitz classes of functions and distributions in $E_{n}, 487$.

Tamura, Takayuki. Examples of direct product of semigroups or groupoids, 419. Minimal commutative divisible semigroups, 713.

Tillmann, H. G. Vector-valued distributions and the spectral theorem for selfadjoint operators in Hilbert space, 67.

Topping, D. M. Vector lattices of self-adjoint operators, 251.

Vasquez, A. T. See Charlap, L. S.

Ward, H. N. On Ree's series of simple groups, 113.

Watanabe, Takesi. See Kunita, Hiroshi.

Whaples, G. See Grant, K.

Whyburn, G. T. Generic and related mappings, 757.

Widder, D. V. Positive solutions of the heat equation, 111.

Wilansky, Albert and Zeller, Karl. A biorthogonal system which is not a Toeplitz basis, 725.

Yang, C. T. The triangulability of the orbit space of a differentiable transformation group, 405.

Yoshida, Reikichi. On some semi-groups, 369.

Zeller, Karl. See Wilansky, Albert.

\section{RESEARCH PROBLEMS}

Balakrishnan, A. V. Geometry, 737.

Bellman, Richard. Algebra, 41.

Analysis, 333.

Asymptotic behavior of functional equations, 689.

Degrees of noncommutativity, 446. 
Existence of closed subsystems, 689.

Hammer, Joseph. Matrix theory, 41.

Mullin, A. A. Recursive function theory, 737.

Some related number-theoretic functions, 446.

Slater, M. Number theory, 333.

Srivastava, R. S. L. Function theory, 737.

Taussky, O. Matrix commutators of higher order, 738.

\section{BOOK REVIEWS}

Antosiewicz, H. A. See LaSalle, Joseph.

Furstenberg, H. Stationary processes and prediction theory. P. Masani, 195.

Gerretsen, John. See Sansone, Giovanni.

Green, L. W. See Rinow, Willi.

Hochschild, G. See Jacobson, Nathan.

Hoffman, Kenneth. Banach spaces of analytic functions. H. Mirkil, 687.

Jacobson, Nathan. Lie algebras. G. Hochschild, 37.

Kappos, D. A. Strukturtheorie der Wahrscheinlichkeitsfelder und -räume. Dorothy Maharam, 207.

Köthe, Gottfried. Topologische lineare Räume. Bd. 1. Leopoldo Nachbin, 733.

LaSalle, Joseph and Lefschetz, Solomon. Stability by Liapunov's direct method with applications. H. A. Antosiewicz, 209.

Lefschetz, Solomon. See LaSalle, Joseph

Lorch, E. R. See Naimark, M.

Maharam, Dorothy. See Kappos, D. A.

Masani, P. See Furstenberg, $H$.

Mirkil, H. See Hoffman, Kenneth.

Morgenstern D. and Szab6, I. Vorlesungen über theoretische Mechanik. C. Truesdell, 330.

Nachbin, Leopoldo. See Köthe, Gottfried.

Naimark, M. Normed rings. E. R. Lorch, 193.

Rinow, Willi. Die innere Geometrie der metrischen Räume. L. W. Green, 210.

Sansone, Giovanni and Gerretsen, Johan. Lectures on the theory of functions of a complex variable. Vol. 1. Allen Shields, 39.

Shields, Allen. See Sansone, Giovanni.

Truesdell, C. See Morgenstern, D.

\section{REPORTS OF MEETINGS AND MISCELLANEOUS ARTICLES}

Council and Board of Trustees-1962, 124.

Green, J. W. and Pierce, R. S. Reports of Meetings of the American Mathematical Society: The Summer Meeting in Boulder, 739; The Annual Meeting in Berkeley, 334.

Green, J. W. and Pitcher, Everett. Reports of Meetings of the American Mathematical Society: The April Meeting in New York, 448.

Huff, G. B. Reports of the Meetings of the American Mathematical Society: The November Meeting in Tallahassee, 43.

Meder, A. E., Jr. Report of the Treasurer, 213.

Montgomery, Deane. Oswald Veblen, 26.

Pierce, R. S. Reports of Meetings of the American Mathematical Society: The November Meeting in Los Angeles, 44; The April Meeting in New Mexico, 464; The June Meeting in Bellingham, 690. 
See Green, J. W.

Pitcher, Everett. Reports of Meetings of the American Mathematical Society: The October Meeting in Hanover, 42; The February Meeting in New York, 341.

Youngs, J. W. T. Reports of Meetings of the American Mathematical Society: The November Meeting in Evanston, 45; The April Meeting in Chicago, 463.

\section{INVITED ADDRESSES}

Atiyah, M. F., 42.

Baily, W. L., Jr., 463.

On the theory of automorphic functions and the problem of moduli, 727.

Bishop, Errett, 690.

de Branges, Louis, 744.

Brauer, Richard. On finite groups and their characters, 125.

Burgers, J. M. On the emergence of patterns of order, 1.

Gehring, F. W. Quasiconformal mappings in space, 146.

Heins, A. E., 45.

Helgason, Sigurdur, 739, 744.

Ito, Noboru. Transitive permutation groups of degree $p=2 q+1, p$ and $q$ being prime numbers, 165.

Kuranishi, Masatake, 341.

McShane, E. J., 334.

Integrals devised for special purposes, 597.

Mackey, G. W. Infinite-dimensional group representations, 628.

MacLane, G. R., 463.

MacLane, Saunders, 739.

MacNerney, J. S., 43.

A linear initial-value problem, 314.

Rauch, H. E., 448.

Royden, H. L. Function algebras, 281.

Sario, Leo. Complex analytic mappings, 439.

Scott, Dana, 44.

Shannon, C. E., 334.

Shapiro, Victor, 44.

Shimura, G., 448.

Smale, Stephen. A survey of some recent developments in differential topology, 131.

Sobolev, Sergei, 448.

Stein, E. M., 334.

Steinberg, Robert, 464.

Sternberg, Shlomo, 739, 744.

Thompson, John, 334.

Vaught, R. L. Models of complete theories, 299. 